\title{
A SATELLITE MOBILE SYSTEM INTEGRATED \\ WITH THE TERRESTRIAL CELLULAR NETWORK
}

\author{
Enrico DEL RE \\ Università di Firenze - Dipartimento di Ingegneria Elettronica \\ Via S. Marta, 3 - 50139 Firenze (Italy)
}

\section{Abstract}

Cellular systems are an efficient solution based on terrestrial infrastructures to satisfy the needs of future users of land mobile communications. To achieve a faster wide area coverage, the integration of a satellite system with the terrestrial cellular network is an interesting approach, provided that as far as possible the same protocols can be used in the two environments. Moreover, this concept can be straightforwardly extended to provide one integrated system for all mobile communications (Land/Aeronautical/Maritime, LAM system). This paper proposes an integrated satellitecellular system with reference to the Pan European digital cellular network recently standardized. The integrated system uses the same protocols except the RF part of the physical layer and the synchronization strategy in the access phase.

\section{INTRODUCTION}

Digital cellular systems are well suitable to satisfy the present and projected user needs for land mobile communications. They are based exclusively on terrestrial infrastructures. They do not take into consideration any possible role of an integrated satellite system. On the other hand, mobile communications are one of the most attractive applications of communications satellites for their inherent capability of covering wide regions on land, on sea and in the air without a capillary terrestrial infrastructure network and for their flexibility able to cope with variable user and traffic demands. Therefore, an integrated satellitecellular system appears an interesting solution, provided that the same protocols can be used as far as possible in the two environments. This approach offers the advantage of a wider and quicker area coverage. More importantly it could provide one integrated system for all mobile communications, i.e. a Land-AeronauticalMaritime (LAM) system.

The concept of an integrated satellitecellular system has received attention recently in organizations like the European Space Agency [2] in Europe and AT\&T in the USA [7]. In Europe the CEPT Study Group on Mobiles has defined the specifications of the new generation of a Pan European Cellular Mobile Communication System (the so called GSM System) [1]. This paper, summarizing the results of [2], presents the structure and the performance of a satellite system integrated with the GSM network.

\section{GSM SYSTEM ARCHITECTURE}

The main building blocks of the GSM system, which are indicated in Fig. 1, are [1]:

- the Mobile Station (MS)

- the Base Station (BS), to which the MS is connected through a radio link; a BS controls a number of cells.

- the Mobile-services Switching Centre (MSC), to which the BSs are connected through a terrestrial link; the MSC is the interface between the fixed terrestrial network and the GSM network.

The main specifications proposed for the GSM system are the following:

- Digital transmission;

- Frequency bandwidths: 25+25 $\mathrm{MHz}$ (890-915 and 935-960 $\mathrm{MHz}$ bands);

- Carrier spacing: $200 \mathrm{kHz}$, providing 125 available carriers in $25 \mathrm{MHz}$ bandwidth;

- Frequency reuse: 9 groups of carriers for the cellular operation;

- Multiple access: TDMA with 8 channels per carrier with the format of Fig. 2 .

\section{INTEGRATED SATELLITE/GSM SYSTEM}

The integration of the satellite and GSM networks (Fig. 1) requires the use of the same protocols apart the RF sections.

The operation of the MS should be to select at any instant of time which one of the two links (satellite or GSM) has better characteristics, for example comparing the levels of the signals received from the satellite and from the GSM base station. This operation is an extension of functions already existing in the GSM MS [1].

The main procedures from a network point of view for the integrated satellite/GSM system are: a) location registration, b) call

\subsection{1.}




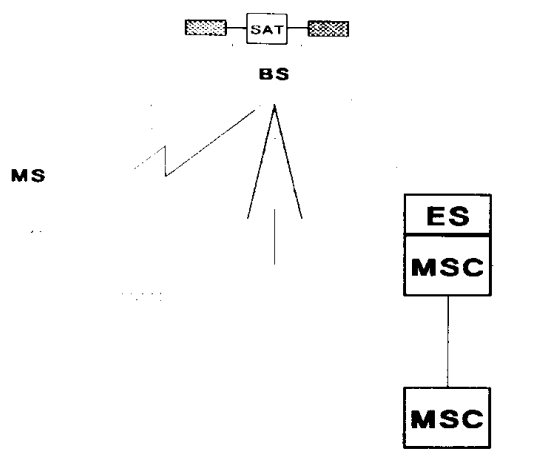

Fig. 1 - Integrated setellite-oSM system

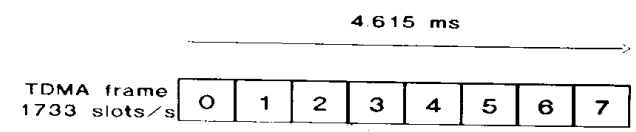

PSTN

etc

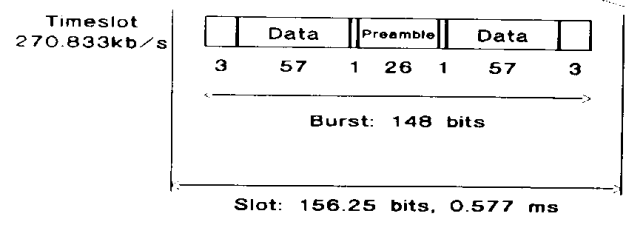

Fig 2 - Basic burst and TDMA frame structures handling, c) handover, d) synchronization of the TDMA access. One interesting outcome of the analysis reported in [2] is that the same protocols of the GSM network can be used to implement the first three procedures and that the Earth Stations (ESS) of the satellite system play the role of the MSCs and their associated BSs. Differently, the synchronization procedure requires some adaptations.

\subsection{Synchronization strategy}

For the synchronization strategy of the TDMA access both in the GSM system and in the satellite system we must distinguish two states (modes of operation):

i) normal state, which is present during the call already in progress;

ii) access state, which is present in the call setup request phase, e.g. at the start of a new connection or at handover.

i) Normal State

The GSM procedure during normal operations (e.g. during the call in progress) can be extended to the integrated system. The delay from the MS is continuously monitored on board or at an ES, communicating its variations to the MS, that accordingly updates the transmission time instant, so that the transmitted burst is always correctly positioned within the assigned timeslot $[2,8]$.

ii) Access State

In the GSM access state, the MS sends an Access Burst (AB) of 88 bits $(0.325 \mathrm{~ms})$ with a guard time of 68.25 bits $(0.252 \mathrm{~ms})$. In this state the MS has no information on its position relative to the BS, but the guard time is sufficient to allow for a maximum cell radius of about $35 \mathrm{~km}$.

In a satelite system with multiple spot beams we have to cope with spot radius of about $1000 \mathrm{~km}$. A guard time of $7.2 \mathrm{~ms}$ is therefore necessary, corresponding to 1950 bits. In this case one slot is not sufficient and even one frame $(4.615 \mathrm{~ms})$ is shorter than the necessary guard time. An even longer guard time is necessary for a satellite with a single beam covering Europe (Eurobeam). A different approach is required in the access state of the satellite system. Two alternatives are possible.

1) Carrier reservation for the access state

We shall consider here the case of a satellite system with multiple spot beams. The extension of the synchronization procedure to a Eurobeam system is straightforward.

A complete carrier is reserved for the access procedure in the satelite system for each spot (Access Carrier). This Access Carrier is subdivided in Access Timeslots corresponding to two GSM frames, whose duration of $9.23 \mathrm{~ms}$ is sufficient for the necessary guard time and the Access Burst duration. The Access Timeslots are simply derived by the MS terminal from the received timing signals from the satellite, for example detecting the start of even (or odd) frames. Successively, the satellite system receiving the Access Burst measures (on board or at an earth station) the estimated round trip delay (satellite-MS-satellite) and sends the corresponding information to the MS, that can thus start using the burst format of the GSM system.

2) Cooperation with a positioning system

If an autonomous positioning system is available at the MS, the mobile position can be known with a sufficient accuracy (i.e. within a radius of about $35 \mathrm{~km}$ ) to allow for the use of the same GSM procedure in the access state.

\subsubsection{Performance Analysis of the Satellite Access Procedure with Carrier Reservation}

The Access Burst is transmitted by the MS after the detection of the start of the even (or odd) frames. The starts of transmission of the Access Burst are Iikely to be random points within the two frame period of the

7.5.2. 
Access Timeslot. Therefore, as a first order approximation, we can assume the Aloha model to represent this transmission procedure.

From the GSM Recommendations a mean value of 2.4 BHCA, corresponding to $0.66610^{-3}$ attempt/s, can be assumed for each MS. The Access Burst duration is $0.325 \mathrm{~ms}$. Thus the Access Traffic/Subscriber is $0.216 \mu \mathrm{Erl} / \mathrm{Sub}$.

The maximum throughput of an Aloha system is 0.18 [3]. Therefore in the Access state the satelite system has the capacity of $0.18 / 0.21610^{6} \approx 830,000$ subscribers.

The average time required to successfully transmit the Access Burst is given by

$$
T=d+b+R_{x} d, \quad \text { where }
$$

d is the collision detection delay,

$\mathrm{b}$ is the burst transmission time (0.325 ms), $R_{\mathbf{x}}$ is the average number of retransmissions.

As in practice a satellite system will operate with a throughput less than 0.1 , the average number of retransmissions can be estimated to be less than 0.25 [3]. The approximate value of $d$ is $260 \mathrm{~ms}$ (round trip delay measured on board) or 520ms (measured at an ES). Therefore the average delay time is in the two cases, respectively

$$
\mathrm{T} \approx 325 \mathrm{~ms} \text { or } 650 \mathrm{~ms}
$$

\subsection{Satellite configurations}

Different scenarios can be envisaged for satelite system integrated with the terrestrial GSM network: transparent satellite, satellite with limited on board processing and satellite with enhanced on board processing.

\subsubsection{Transparent satellite}

A number of different architectures can be envisaged. They all assume a single beam for the fixed Earth Stations (ESs) [2].

A1 - Only one ES for the whole system; a single Eurobeam for mobiles.

A2 - Only one ES; multiple spot beams for mobiles.

In both solutions the $\mathrm{ES}$ is the unique interface with the terrestrial GSM network: aII the traffic to/from mobiles that uses the satellite passes through this station, whose role is similar to a GSM Base station (with its associated MSC) that now controls all the spot beams instead of the cells. Very long terrestrial tails from the ES to the end users are present that may not be acceptable for the management problems associated with the presence of the different Administrations in Europe. In addition solution Al car provide a limited capacity.

BI - Multiple ESs in the single beam (e.g. as many as the number of spots for mobiles); multiple spot beams for mobiles.

This solution assumes that the traffic directed to mobiles of a given spot pass through the ES located in (or closest to) that spot. The satellite simply acts as a repeater connecting a MS in a given spot with its closest $E S$, which again performs the functions of a GSM BS now controlling only one spot. Long terrestrial tails are present for traffic directed outside the spot of the MS. However, due to the traffic distribution $[2,6]$, they are likely to occur infrequently and may become acceptable.

B2 - Multiple ESs; multiple spot beams for mobiles.

To reduce the terrestrial tails this solution assumes that the mobiles are connected to the ES closest to the end user. To simplify the constraints on the synchronization procedure among all the ESs accessing the satellite in TDMA, a fixed number of carriers can be assigned to each combination of ES-spot. This fixed assignment may lead to an inefficient system if a small number of carriers is available through the satellite.

B3 - Multiple ESs; multiple spot beams for mobiles.

The inefficiency of solution B2 can be eliminated using an unconstrained TDMA access from ES to MS: this however increases the complexity of the ES [2].

In any configuration the transparent satellite alternative simply substitutes the GSM network link MS-BS-MSC with the satellite Iink MS-ES. All the network functions remain under the responsibility of the terrestrial GSM network.

The transparent satellite allows for the communication with mobiles in areas covered by the satellite and not by the GSM network, but, for practical reasons, it cannot be used for direct MS-to-MS communication through the satellite.

\subsubsection{Satellite with on board processing}

Two solutions can be considered.

C - In addition to multiple ESs and multiple spot beams for mobiles, we assume a satellite with a base-band on board processing in charge of switching the calls. All the functions required for call routing and system management can be provided on ground at a control center through a double-hop connection.

The uplinks and downlinks between the satelite and the MSs are in TDMA on multiple carriers, as in the GSM system. The uplinks and downlinks between the satellite and the fixed ESS are in TDMA on a high-rate unique carrier. The on board switching function provides for the correct connections between the links on the ES side and the links on the

7.5.3. 
MS side. This technical solution simplifies the complexity of the ES. It also provides end-to-end connections with relatively short terrestrial tails and allows for direct MSto-Ms communications through the satellite.

Moreover a reallocation of resources (channels) among the various ESs to improve the system efficiency is now possible through the ground control station and it is very easy to increase the number of fixed ESs as required.

D - With respect to $C$, this solution adds other processing capabilities on board in order to avoid the double-hop connection for some signalling functions required by the integrated system. This is of course the most powerful system with optimized performances. At the moment it can be considered as a long term solution, whose feasibility and convenience will depend on the advances of the on board processing technology and the integrated system operational requirements.

\section{SATELLITE SYSTEM PERFORMANCE}

The performance of a satellite system integrated with the terrestrial GSM network is considered referring to the present bandwidth allocation for a land mobile satellite system [4]. The different alternatives described in sect. 3 will be evaluated with the following as sumptions :

- Bandwidth: $7 \mathrm{MHz}$ (full duplex) at L band;

- Carrier spacing: $200 \mathrm{kHz}$

- Beam configurations: Eurobeam, 7 spot beams, 12 spot beams (Figs. 3 and 4)

- Frequency reuse (spot beams cases): 3 groups of carriers

- Multiple access: TDMA with 8 channels per carrier, with the same format as the GSM system;

- Subscriber traffic intensity: $20 \mathrm{mEr} 1 / \mathrm{sub}$.

- The system capacity is assumed to be limited only by the number of available channels to/from mobiles: in other words the link to/from the ESs is assumed to have the necessary bandwidth (operating at $\mathrm{Ka}$ or $\mathrm{Ku}$ band) and a full connectivity is assumed on board the satelite.

Table 1 shows the performance of each satellite configuration. Greater details are reported in [2]. The number of subscribers refers to a blocking probability (grade of service) of $2 \%$, according to the Erlang-B formula [5]. The last column (system R) gives the maximum capacity of the system when bandwidth reallocation among the spot beams for mobiles is employed: the result refers to the Iimiting case of maximum reallocation of the available channels. The result for the system alternative B2 has been obtained as suming a traffic distribution of $95 \%$ in the same spot and $5 \%$ uniformly distributed among the other spots [6].

Peak power indicates the RF power required at the MS during the burst transmission in the TDMA frame. The mean $R F$ power required at the mobile transmitter is therefore approximately $1 / 8$ of the peak power.

CCITT Recommendation $G .114$ suggests a maximum delay for speech of $400 \mathrm{~ms}$. GSM Recommendations assume a delay of approximately $90 \mathrm{~ms}$ for the speech transmission between the MS and the BS (or the MSC), including signal processing and transmission. With $260 \mathrm{~ms}$ of signal delay through the satellite, CCITT Recommendation can be satisfied only avoiding a double $90 \mathrm{~ms}$ contribution to the overal1 delay. As shown in [2], only configurations B2, B3, C, D and $R$ can avoid the double contribution.

\section{CONCLUSIONS}

The results $[2,8]$ suggest some of the proposed alternatives as feasible solutions for the integration of a satellite system with the terrestrial GSM network for mobile

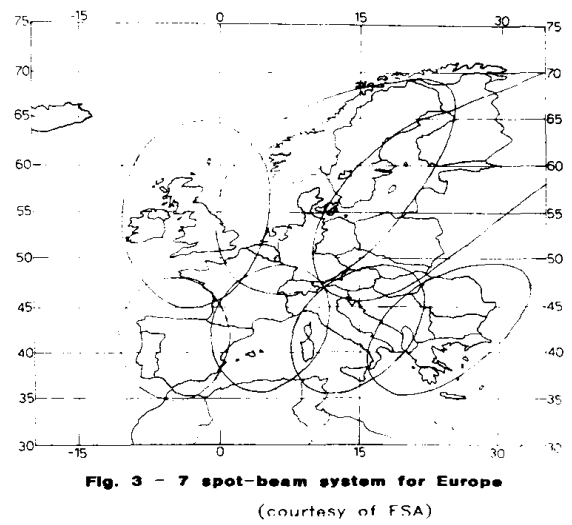

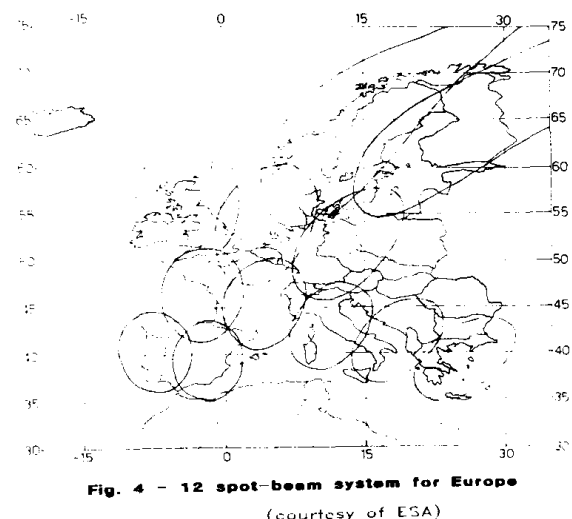

(courtosy of ESA)

\subsection{4.}


SYSTEM

DIRECT MS-to-MS COMMUNICATION

(N=No, $Y=$ Yes )

\section{NO. OF SUBSCRIBERS \\ Eurobeam \\ 7 spot beams \\ 12 spot beams \\ MS PEAK RF POWER (W) \\ Eurobeam \\ 7 spot beams \\ 12 spot beams}

SPEECH DELAY (ms)
A1

A2, B1

B3, C, D

B2

$\mathbf{N}$

$\mathbf{N}$

\author{
$\mathbf{N}, \mathbf{N} \quad \mathbf{N}, \mathbf{Y}, \mathbf{Y}$
}

12888

$\begin{array}{llcc}24045 & 24045 & 8729 & 33885 \\ 41220 & 41220 & -- & 56475\end{array}$

60
$41220 \quad 41220$

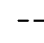

56475

$\begin{array}{cccc}6 & 6 & 6 & 6 \\ 3 & 3 & 3 & 3 \\ 440 & 350 & 350 & 350\end{array}$

Table 1 - Performance of satellite configurations

communications.

The main technical advantages are:

- only one mobile terminal is required for the satellite and the GSM systems, making it attractive for its potential low cost;

- the ESs of the satellite system are similar to the GSM BS and MSC switching centres: this avoids an expensive development for the ESs that can be adapted from the GSM BS and MSC stations and may even be colocated with some of them saving the common parts.

The main operational benefits that could be achieved are the extension of the mobile services with the GSM standards to:

- not yet covered Western Europe areas;

- Eastern Europe and North Africa areas;

- aeronautical and maritime mobiles, so that one integrated land/aeronautical/maritime system (LAM system) could be conceived for all mobiles.

In particular the architectures A2, B1 and B3 would allow to deploy in a short time a transparent satellite payload for a system integrated with the terrestrial GSM network. These solutions have some limitations and drawbacks and a reallocation strategy is more difficult to implement. Nevertheless they provide a feasible system with a sufficient capacity for the start-up phase of the service.

Architectures $C$ and $D$ are much more efficient, avoiding all the limitations and drawbacks of the previous solutions at the expense of a more complex on board payload. Maybe their selection is more easily justified if we increase the number of spot beams and use higher frequency bands (instead of $\mathrm{L}$ band): the consequent increase of the system capacity in terms of total number of available channels can justify a more complex on board payload.
Acknowledgment

This study has been carried out during a period of leave of the author at the EUROPEAN SPACE RESEARCH AND TECHNOLOGY CENTRE of the EUROPEAN SPACE AGENCY, Noordwijk, The Netherlands.

\section{REFERENCES}

[1] GSM Recommendations, CEPT Study Group on Mobiles, CEPT/CCH/GSM/PN, February 1988 .

[2] E. Del Re, "Satellite systems integrated with the terrestrial cellular network for mobile communications", European Space Agency, STR 228, March 1989.

[3] M. Schwartz, Telecommunication networks, Addison-Wesley Pub. Co., Reading, MA, 1987.

[4] World Administrative Radio Conference for the Mobile Services, Geneva, 14 Sept.-16 oct. 1987.

[5] L. Kleinrock, Queueing Systems, John Wiley \& Sons, New York, 1975.

[6] J. M. Casas, P. Bartholome', "Some aspects of the implementation of an integrated space/terrestrial network for Europe", in E. Del Re, P. Bartholome', P.P. Nuspl, Eds., Satellite Integrated Communications Networks, North-Holland, Amsterdam, The Netherlands, 1988.

[7] E.S.K. Chien, J.A. Marinho, J.E. Russell Sr., "Design mobile satellite system architecture as an integral part of the cellular access digital network", Proc. Mobile Satellite Conf., JPL, Pasadena (USA), May $3-5,1988$.

[8] E. Del Re, "An integrated satellitecellular land mobile system for Europe", 8 th Int. Conf. Digital Sat. Comm., Guadeloupe, April 24-28, 1989.

\subsection{5.}

